\title{
ALERT: Um Sistema Multicamadas para a Disseminação de Informações em Redes Veiculares
}

\author{
Yan V. Brandão ${ }^{1}$, Lucas M. de Souza ${ }^{1}$ Thiago S. Gomides $^{1}$ \\ e Daniel L. Guidoni ${ }^{1}$ \\ ${ }^{1}$ Departamento de Ciência da Computação \\ Universidade Federal de São João Del Rei, (UFSJ) - MG - Brasil \\ \{brandao, lmarchisotti,gomides, guidoni\}@ufsj.edu.br
}

\begin{abstract}
The growth in the number of traffic accidents has become a cause for concern in urban centers. As a result of the increase of population in large cities and the increase in the number of vehicles, the accidents impacts may be even greater. In order to reduce these impacts, we present ALERT, a fully distributed algorithm capable of reducing congestion caused by accidents, thus contributing to an efficient urban mobility in smart cities. The algorithm performs the detection of accidents and the dissemination of warning messages in multiple hops. In addition, the system is able to share road traffic information, so vehicles know in advance the conditions of road displacement of nearby segments. Simulation results indicate that the proposed algorithm is capable of reducing the average travel time of the vehicles.
\end{abstract}

Resumo. O crescimento do número de acidentes de trânsito tem se tornado preocupante nos centros urbanos. Em consequência do agrupamento populacional nas grandes cidades e o aumento do número de veículos, os impactos dos acidentes podem ser ainda maiores. Com o propósito de diminuir esses impactos, nós apresentamos o ALERT, um algoritmo totalmente distribuído capaz de reduzir os congestionamentos ocasionados por acidentes, contribuindo para uma mobilidade urbana eficiente em cidades inteligentes. $O$ algoritmo realiza a detecção de acidentes e disseminação de mensagens de alerta em múltiplos saltos. Além disso, o sistema é capaz de realizar o compartilhamento de estimativas de tráfego das vias, para que os veículos saibam previamente das condições de deslocamento dos segmentos próximos. Resultados de simulação indicam que o algoritmo proposto é capaz de reduzir o tempo médio de viagem dos veículos.

\section{Introdução}

O aumento da população, a falta de atenção de motoristas e o excesso de tráfego são fatores que colaboram com o aumento dos acidentes de trânsito, segundo a Organização Mundial da Saúde (OMS) [WHO 2020]. Anualmente, o número de mortes por acidentes de trânsito em todo o mundo ultrapassa 1,3 milhões de pessoas. Além disso, os prejuízos financeiros, os danos à qualidade de vida, as lentidões e paralisações do trânsito, são fatores que aumentam os interesses das discussões entre governos, pesquisadores e ambientalistas. Assim, busca-se soluções capazes de auxiliar e minimizar esses efeitos. Atualmente, o processo de notificação de acidentes é lento e pouco eficiente [Hussain et al. 2019]. Segundo [Hussain et al. 2019], esse processo pode ser dividido em três etapas: (i) Relato físico, onde descreve-se aos serviços de segurança detalhes 
sobre a ocorrência; (ii) Relato via broadcast, etapa de disseminação da informação realizada via rádio ou através de uma unidade GPS móvel e, por fim, (iii) alerta aos motoristas.

Em contrapartida e em busca de maior eficácia na detecção e solução de congestionamentos causados por acidentes, nas últimas duas décadas, a indústria automotiva se baseou em inovação tecnológica como resultado de avanços significativos em tecnologias de computação, comunicação e de armazenamento [Weiner 2010]. Com o avanço da comunicação móvel e com a padronização das redes veiculares, os Sistemas de Transporte Inteligente (em inglês Intelligent Transportation System - ITS) são incentivados com o objetivo de utilizar a comunicação entre veículos para reduzir problemas nos transportes tradicionais em centros urbanos. Assim, populariza-se a utilização das Redes Veiculares (em inglês Veicular Ad Hoc Networks - VANETs), que são redes móveis onde veículos comunicam-se entre si ou com a infraestrutura viária tendo como foco a segurança nas rodovias, a troca de informações sobre o trânsito e entretenimento. Segundo [Weiner 2010], diversas aplicações para VANETs foram desenvolvidas nos últimos anos e, dentre elas, a disseminação de mensagens de segurança.

Com relação a comunicação em redes veiculares, os alertas de segurança possuem maior prioridade em comparação com outros tipos de mensagens devido à sua importância e finalidade de aplicação. Em situações de acidentes de trânsito, a agilidade e eficiência para atingir seu objetivo e confiabilidade, são essenciais. Assim, o envio de mensagens na ocorrência do acidente acelera a disseminação da informação e, consequentemente, a tomada de decisão. Quando a informação é compartilhada em tempo hábil, vidas podem ser poupadas devido a notificação aos serviços de emergência com rapidez. Além disso, destaca-se a redução dos impactos no trânsito da região do acidente por gerar alternativas de novos trajetos para veículos com rota no local.

Nesse contexto, o objetivo deste trabalho é propor o ALERT : Um Sistema Multicamadas para a Disseminação de Informações em Redes Veiculares. O ALERT tem como tarefa alertar os motoristas sobre a ocorrência de acidentes de forma eficiente tendo como consequência o aumento da segurança no trânsito, a construção de uma rede de conhecimento sobre as condições de tráfego e a mitigação de congestionamentos causados por acidentes. A solução proposta foi avaliada e comparada com soluções da literatura e apresenta melhores resultados em termos de tempo de viagem, tempo perdido e da distância percorrida. O restante deste artigo está organizado como segue. A Seção 2 apresenta os principais trabalhos relacionados. A Seção 3 detalha o funcionamento do protocolo proposto. As simulações e os resultados do estudo da avaliação e do desempenho do protocolo são apresentados na Seção 4. Por fim, a Seção 5 apresenta as conclusões e os potenciais trabalhos futuros.

\section{Trabalhos relacionados}

Os sistemas de gerenciamento veicular são comumente classificados em três abordagens em relação à infraestrutura de comunicação, sendo centralizada, descentralizada e distribuída [Ahmed and Gharavi 2018]. Em soluções centralizadas, os veículos recebem rotas alternativas de um servidor central que possui uma visão global das informações de tráfego. Sistemas de Navegação Veicular (em inglês Vehicular Navigation System - VNS), como Google Maps e Waze são exemplos dessas soluções. As soluções distribuídas são baseadas apenas na comunicação entre veículos. Durante o deslocamento, os veículos disseminam e recebem informações sobre as condições de tráfego para estimar localmente 
rotas alternativas [de Souza et al. 2018]. Em soluções descentralizadas, uma infraestrutura auxiliar (Road-Side Units (RSUs) é implantada nas ruas para receber, processar e disseminar informações de tráfego e rotas alternativas aos veículos.

Em [de Souza et al. 2018], os autores realizaram um estudo sobre a eficiência do compartilhamento de informações em sistemas de gerenciamento de trânsito e apresentaram o Preventing traffic congestioN through a fully-Distributed Rerouting Algorithm (PANDORA). O PANDORA é um algoritmo baseado em conteúdo flutuante para melhorar a eficiência geral do tráfego. O algoritmo realiza a disseminação oportunista do conhecimento através de um método de conteúdo flutuante e informações de tráfego localizadas geograficamente em áreas críticas. As áreas críticas definem regiões onde o sistema deve ser executado para reduzir o congestionamento local. Além disso, pesquisas similares também foram realizadas em [Wang et al. 2016] pelos autores, que apresentaram o Next Road Rerouting (NRR), um sistema multiagente para mitigar congestionamentos inesperados de tráfego urbano. O NRR é um algoritmo que auxilia motoristas na ocorrência de eventos inesperados no trânsito, como o acidente entre veículos, realizando re-roteamentos para minimizar os congestionamentos. Contudo, o sistema realiza análise somente da próxima via em questão para a obtenção de novas rotas, o que pode resultar em percursos longos.

Em [Gomides et al. 2019] é apresentado o SGTD: um Sistema de Gerenciamento de Tráfego totalmente Distribuído capaz de classificar e reorganizar o fluxo de veículos para reduzir congestionamentos. Neste trabalho os autores definem níveis de congestionamento para classificar o estado atual das vias. Essa classificação é realizada pelos veículos através de análises momentâneas do estado da via durante o deslocamento. $\mathrm{O}$ algoritmo realiza o compartilhamento das estimativas através de mensagens do tipo Beacon de único salto e, a partir das estimativas recebidas, os veículos realizam o cálculo de novas rotas afim de reduzir os congestionamentos.

\section{ALERT}

Para promover as cidades inteligentes e a melhoria da qualidade do tráfego de forma eficiente, apresentamos o ALERT. Fundamentado na comunicação veículo-paraveículo (V2V), o ALERT é um sistema de gerenciamento de tráfego com mecanismos de coleta e entrega eficiente de conhecimento sobre congestionamentos em cenários de congestionamentos causados por acidentes de trânsito. Assim, cada veículo monitora o seu deslocamento e compartilha os dados coletados com os veículos vizinhos. Deste modo, os veículos são capazes de tomarem decisões de re-roteamentos, otimizando a mobilidade urbana com maior segurança e menor tempo no trânsito. Nesse sentido, o sistema pode ser divido em três camadas, sendo estas: (i) Acidente, (ii) Gerenciamento de Tráfego e (iii) Cidade Inteligente, como apresentado na Figura 1. A camada (i) é responsável por controlar os congestionamentos causados por acidentes de trânsito, gerenciando os bloqueios das vias que podem acontecer de forma parcial ou completa. A camada (ii) realiza o gerenciamento de tráfego devido ao fluxo de veículos. Por fim, a camada (iii) interliga o funcionamento das camadas em (i) e (ii) e define como os veículos e a infraestrutura da cidade se relacionam. Para isso, a estrutura da cidade pode ser representada como apresentado na Definição 1.

Definição 1 (Cidade Inteligente). Considere o ambiente veicular como parte de uma 

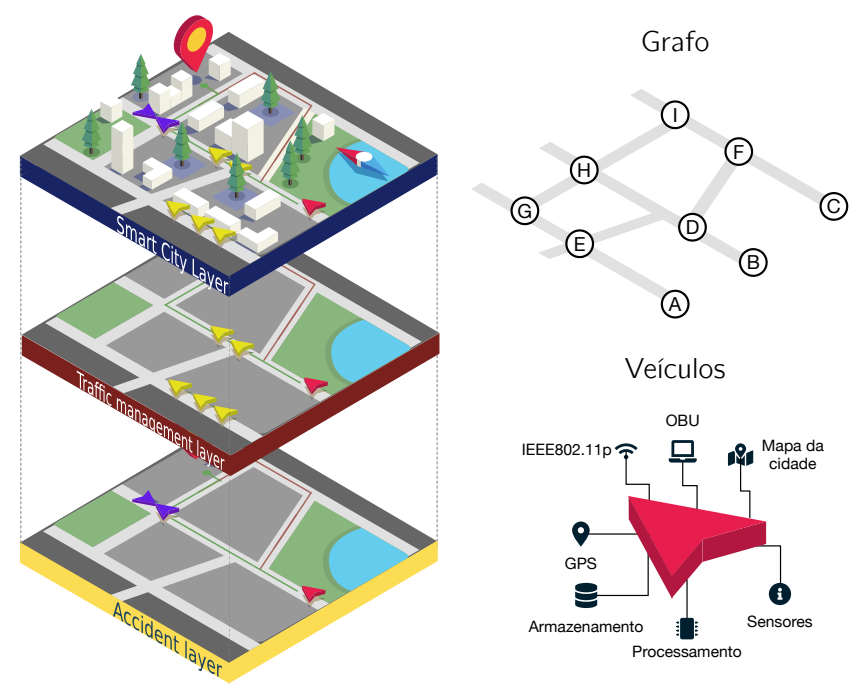

Figura 1. Visão geral do ALERT

cidade inteligente (Figura 1 marcador (Grafo)) e definido por um grafo ponderado $e$ dirigido $G=(V, E)$, onde o conjunto $V=\left\{v_{1}, v_{2}, \ldots, v_{i}\right\}$ representa o conjunto de esquinas do cenário (vértices) e o conjunto $E=\left\{e_{1}, e_{2}, \ldots, e_{i}\right\}$ corresponde as vias/ruas da cidade (arestas). Para cada aresta $e_{i}=\left(v_{1}, v_{2}\right)$, existe um peso associado $w_{i}$ que representa o custo estimado (tempo) para percorrer a aresta, como representado pelo conjunto $W=\left\{w_{1}, w_{2}, \ldots, w_{i}\right\}$.

Nesse ambiente, o sistema ALERT estabelece que cada veículo deve ser equipado com capacidade de processamento, armazenamento, sensoriamento e tecnologias de comunicação veicular, presentes nas Unidades de Bordo (OBU). Além disso, mapa da cidade, receptor do sistema de posicionamento global (GPS), entre outros. As seções seguintes apresentam mais detalhes do funcionamento das camadas (i) e (ii).

\subsection{Camada de Acidentes}

Esta camada é definida por dois comportamentos, sendo estes: (i) a disseminação de alertas aos veículos quando é detectada a ocorrência de um acidente e (ii) a realização do re-roteamento neste cenário, desviando o fluxo dos veículos para uma via com melhores condições de tráfego. Esse processo é definido para minimizar o impacto de um acidente e garantir maior segurança ao trânsito, além de promover maior fluidez na mobilidade urbana. Estabelecemos, para isso, que a detecção de acidente deve ser realizada pelo veículo no momento da colisão através da análise de sinais emitidos por sensores embarcados, como por exemplo, a verificação do acionamento dos Airbags. Nesse sentido, ao verificar a ocorrência de colisão, o veículo deve disseminar alertas através de mensagens de segurança. Cada mensagem de segurança é composta pelo identificador da via onde ocorreu o acidente e o tempo de vida de retransmissão - Time-to-live (TTL).

Devido aos impactos ocasionados pelos acidentes, os veículos envolvidos devem disseminar em múltiplos saltos o compartilhamento das mensagens de segurança. Dessa forma, é possível informar os veículos localizados dentro de uma ampla área de cobertura sobre o acidente e obstrução da via. Assim, veículos que recebem mensagens de segurança devem realizar o cálculo de uma rota alternativa desviando-se da via obstruída 
e, quando necessário, devem também retransmitir a informação para seus vizinhos. A retransmissão de mensagens deve ser realizada de forma eficiente e sustentável, ou seja, a região de cobertura deve ser informada sobre o acidente ocorrido e, ao mesmo tempo, não sobrecarregar a rede de comunicação sem fio. Para isso, é necessário que haja um equilíbrio entre o número de mensagens transmitidas e a perpetuação do alerta, visto que, devido à alta mobilidade, novos veículos podem se aproximar do local do acidente e necessitarão de informações. Assim, o compartilhamento de alertas em múltiplos saltos é realizado em intervalos de tempo que são associados a três níveis de prioridade como apresentado na Figura 2.

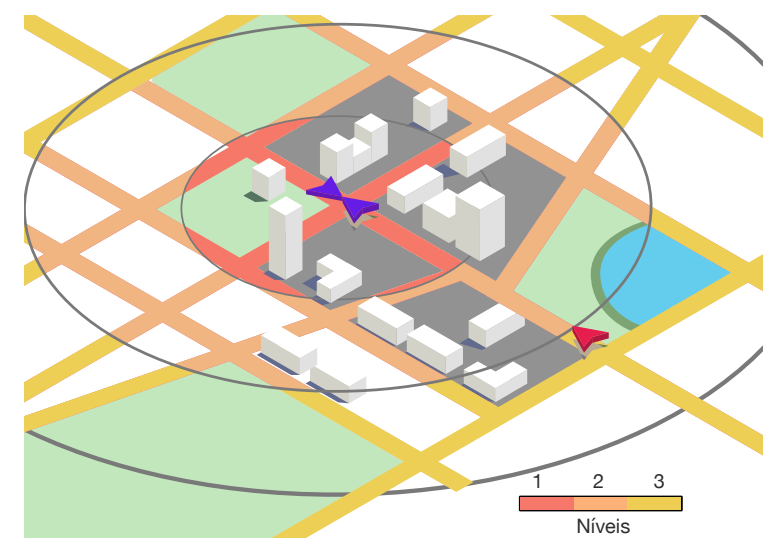

Figura 2. Área de cobertura da camada de acidente

A maior prioridade é direcionada a uma área denominada área crítica que compreende um raio de aproximadamente 300 metros centrado no local do acidente (Nível 1 - região vermelha na Figura 2). Esta área tem maior prioridade e o protocolo deve garantir que os veículos neste local recebam imediatamente a informação do acidente. Para isso, os veículos acidentados disseminam mensagens a cada 15 segundos com TTL $=1$. Na segunda região (Nível 2 - região laranja na Figura 2), os veículos devem receber o aviso sobre o acidente, mas não com a mesma prioridade que na área crítica. Para isso, a disseminação de mensagens é realizada a cada 30 s e com TTL $=2$, atingindo a área dentro de um raio de no máximo 600 metros do acidente. Por fim, para o terceiro nível (Nível 3 - região em amarelo na Figura 2), a informação é direcionada para uma área superior ao raio de 600 metros do local do acidente, mas dentro da região de cobertura. Assim, como esta região é de menor prioridade, a frequência de atualização também é menor. Para atingir esta região, o veículo acidentado dissemina a mensagem a cada 60s com TTL $=5$. Assim, a região de cobertura do acidente foi definida com no máximo 5 vezes o raio de comunicação do veículo.

\subsection{Camada de Trânsito}

A camada de trânsito atua na solução de congestionamentos decorrentes da sobrecarga das vias ou outras situações que não causam o bloqueio total da via, mas ocasionam tráfego lento e paradas recorrentes. Como os veículos podem ser re-roteados através da camada de acidentes, algumas vias podem ser sobrecarregadas, contribuindo para o surgimento de novos congestionamentos em outros pontos da cidade.

Durante o percurso, os veículos realizam a análise do tempo de viagem em cada via. Sempre que o veículo detecta que a via está congestionada, ele realiza o compartilha- 
mento do tempo de viagem verificado através de mensagens do tipo beacon, de forma a atualizar os veículos em sua vizinhança e manter o conhecimento das condições de tráfego distribuído. Para isso, mensagens do tipo beacon são transmitidas em broadcast em único salto, ou seja, não são retransmitidas pelos veículos que a recebem. Assim, durante a viagem os veículos recebem informações sobre as condições de tráfego somente das vias em sua proximidade. Ao receber um beacon, o veículo atualiza sua base de dados para posteriormente buscar por rotas alternativas. A busca por rotas alternativas é realizada pelo veículo somente ao se aproximar de interseções, pois possibilita que o veículo absorva maior quantidade de informações sobre as condições de tráfego das vias antes de buscar por rotas alternativas melhores que a atual. O veículo realiza o cálculo do caminho de menor custo entre a via atual até seu destino final, utilizando o algoritmo de Dijkstra. Caso o custo da rota encontrada seja menor que sua rota atual, ele realiza o re-roteamento para a nova rota. Detalhes de como é realizada a estimativa de tráfego nas vias pode ser encontrada em [Gomides et al. 2019].

\section{Análise dos resultados}

Esta seção avalia o desempenho da solução proposta considerando as ferramentas utilizadas, o cenário de simulação e os resultados obtidos. Para isso, a mobilidade dos veículos foi realizada através da ferramenta SUMO 0.25 [Lopez et al. 2018] e a comunicação veicular foi realizada utilizando o simulador de eventos discretos OMNET++ 5.1.1 [Varga and Hornig 2008] em conjunto do framework de comunicação veicular Veins 4.6 [Sommer et al. 2011]. Veins implementa o padrão IEEE 802.11p utilizado em redes veiculares. As simulações foram realizadas na região central do mapa da cidade de Portland, EUA, sob uma região de $17 \mathrm{~km}^{2}$. Cada veículo no cenário possui um par origem-destino definidos de forma aleatória e o número de veículos varia entre os valores $850,1700,2550,4250,3400,5100$ e 5950, onde o protocolo é avaliado considerando diversas densidades veiculares. Em una análise preliminar do tráfego de veículos, foi verificado qual rua do mapa utilizado possui, em média, o maior movimento de veículos. No instante $t=100 \mathrm{~s}$ um acidente com duração de $1500 \mathrm{~s}$ acontece na rua com maior tráfego de veículos e com obstrução total do tráfego. Foram realizadas 33 simulações para cada número de veículos no mapa, com um intervalo de confiança de $95 \%$.

A figura 3 apresenta os resultados obtidos durante as avaliações de tráfego considerando as estratégias Original (tráfego sem re-roteamento) e as abordagens da literatura PANDORA e NRR. A Figura 3(a) apresenta o tempo médio de viagem verificado para cada algoritmo, onde no eixo x temos a densidade veicular e no eixo y o tempo de viagem em segundos. Destaca-se que a solução proposta possui melhores resultados em relação aos tempos de viagem acima de 3400 veículos. Entretanto, com o aumento do número de veículos a diferença é mais evidente. Considerando 1700 veículos, o tempo de viagem verificado pelo ALERT é 14\% menor em relação ao obtido pelo Original. Em comparação com a solução NRR, o ALERT obteve o tempo médio de viagem 5\% superior. Considerando 5950 veículos, o ALERT obteve o menor tempo médio de viagem entre as soluções apresentadas, sendo este 51\%, 64\% e 77\% inferior em relação ao Original, PANDORA e NRR respectivamente. Assim, através dos resultados obtidos, observa-se que dentre as soluções abordadas neste trabalho, o ALERT é o algoritmo mais eficiente para gerenciar o trânsito em cenários mais congestionados.

Figura 3(b) apresenta a distância média percorrida pelos veículos. O eixo x apre- 


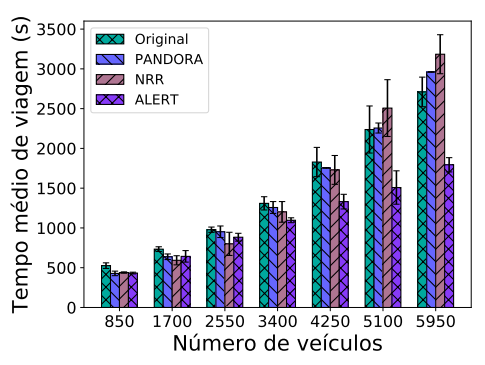

(a) Tempo médio de viagem

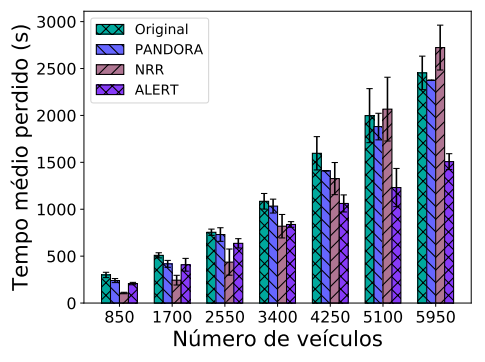

(c) Tempo médio perdido

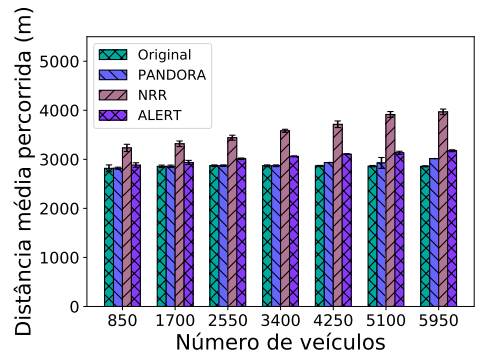

(b) Distância média percorrida

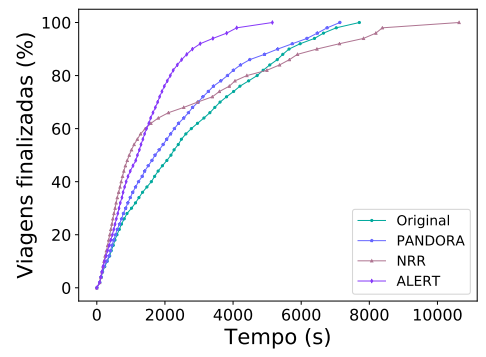

(d) Término das viagens

Figura 3. Resultados obtidos

senta o número de veículos e o eixo y apresenta a distância média percorrida em metros. A distância média percorrida verificada pela solução Original representa o valor ótimo, onde os veículos viajam no caminho mais curto dado o par origem-destino e as rotas não são recalculadas. Considerando 5950 veículos, onde ocorre a maior diferença entre as distâncias percorridas, foi verificado que o ALERT, PANDORA e NRR percorrem em média distâncias de $11 \%$, 5\% e 38\% maiores em relação ao Original, respectivamente. O grande impacto na distância percorrida verificada pelo algoritmo NRR se deve ao fato de o mesmo não considerar o cálculo completo de novas rotas até o destino final, o que ocasiona escolha de novas rotas de distância significativamente maiores. O tempo médio parados em trânsito é apresentado pela Figura 3(c). Destaca-se que o ALERT apresenta maior eficiência em relação as demais soluções nos cenários com maiores densidades de veículos. Considerando 4250 veículos, o tempo médio perdido verificado pelo ALERT é 33\%, 24\% e 19\% menor em relação ao Original, PANDORA e NRR, respectivamente.

Por fim, a Figura 3(d) apresenta o percentual acumulado de veículos que finalizaram as viagens em relação ao tempo. Nesse sentido, no eixo x é apresentado o tempo de viagem em segundos e no eixo y é apresentado a porcentagem acumulada de viagens finalizadas. Dessa forma, considerando os primeiros $60 \%$ dos veículos que chegaram aos seus destinos, o NRR é o algoritmo mais eficiente. A partir deste ponto, a solução proposta foi mais eficiente que os demais algoritmos, onde todos os veículos finalizaram as viagens em 5154s, enquanto o Original, PANDORA e NRR finalizaram com $7707 \mathrm{~s}$, 7134 s e 10635 s respectivamente.

\section{Conclusão}

Este trabalho apresenta uma solução para a comunicação de alertas de acidentes de trânsito e re-roteamento de veículos. O modelo proposto realiza verificação de ocorrência de acidentes e sugere novas rotas para os veículos que percorreriam a via onde o acidente 
ocorreu. Assim, a proposta possui como objetivo reduzir e garantir que os congestionamentos não sejam deslocados para outras regiões da cidade. Dessa forma, os resultados de simulações demonstram o desempenho da nossa solução comparada à estratégia Original e às soluções da literatura NRR e PANDORA. Os resultados mostraram que a solução proposta consegue reduzir o tempo do trajeto e o tempo médio parado em trânsito com pouco impacto na distância percorrida. Como trabalhos futuros, pretende-se a avaliação da solução em cenários com múltiplos acidentes e utilizando traces realísticos.

\section{Agradecimentos}

Os autores agradecem a Fundação de Amparo à Pesquisa do Estado de Minas Gerais (FAPEMIG) sob a concessão n. ${ }^{\circ}$ : APQ-03120-17 e ao Conselho Nacional de Desenvolvimento Científico e Tecnológico (CNPq) sob a concessão n. ${ }^{\circ}:$ 150545/2018-5. O presente trabalho foi realizado com apoio da Coordenação de Aperfeiçoamento de Pessoal de Nível Superior - Brasil (CAPES) - Código de Financiamento 001.

\section{Referências}

Ahmed, E. and Gharavi, H. (2018). Cooperative vehicular networking: A survey. IEEE Transactions on Intelligent Transportation Systems, 19(3):996-1014.

de Souza, A. M., Pedrosa, L. L. C., Botega, L. C., and Villas, L. (2018). Itssafe: An intelligent transportation system for improving safety and traffic efficiency. In 2018 IEEE 87th Vehicular Technology Conference (VTC Spring), pages 1-7.

Gomides, T., Fernandes, M., Souza, P., and Guidoni, D. (2019). Sgtd: Sistema de gerenciamento de tráfego distribuído para redes veiculares.

Hussain, R., Hussain, F., and Zeadally, S. (2019). Integration of vanet and 5g security: A review of design and implementation issues. Future Generation Computer Systems, $101: 843-864$.

Lopez, P. A., Behrisch, M., Bieker-Walz, L., Erdmann, J., Flötteröd, Y.-P., Hilbrich, R., Lücken, L., Rummel, J., Wagner, P., and Wießner, E. (2018). Microscopic traffic simulation using sumo. In The 21st IEEE International Conference on Intelligent Transportation Systems. IEEE.

Sommer, C., German, R., and Dressler, F. (2011). Bidirectionally Coupled Network and Road Traffic Simulation for Improved IVC Analysis. IEEE Transactions on Mobile Computing, 10(1):3-15.

Varga, A. and Hornig, R. (2008). An overview of the omnet++ simulation environment. In Proceedings of the 1st International Conference on Simulation Tools and Techniques for Communications, Networks and Systems \& Workshops, Simutools '08.

Wang, S., Djahel, S., Zhang, Z., and McManis, J. (2016). Next road rerouting: A multiagent system for mitigating unexpected urban traffic congestion. IEEE Transactions on Intelligent Transportation Systems, 17(10):2888-2899.

Weiner, S. (2010). Feasibility of a 802.11 vanet based car accident alert system. Northeastern University.

WHO (2020). Road traffic injuries. https://www.who.int/news-room/ fact-sheets/detail/road-traffic-injuries. 